\title{
O Uso do Robomind no Processso de Aprendizagem de Programação: Uma Revisão Sistemática da Literatura
}

\author{
Leonardo S. Silva ${ }^{1}$, Elmano Ramalho Cavalcanti ${ }^{2}$ \\ ${ }^{1}$ Instituto Federal de Pernambuco \\ - Garanhuns - PE - Brasil \\ ${ }^{2}$ Instituto Federal de Pernambuco \\ - Jaboatão dos Guararapes - PE - Brasil \\ leonardo.silva@garanhuns.ifpe.edu.br, elmano.cavalcanti@jaboatao.ifpe.edu.br
}

\begin{abstract}
Robomind is an educational technology supported by playful and pedagogical resources to assist programming learning. Summarizing the academic evidence already published on the use of this software is essential to assess its effectiveness. A systematic literature review was performed, and found that students who used Robomind showed greater motivation with their studies, interest in the area of computing and lower dropout. On the other hand, the learning gains were conflicting. Finally, the scientific and statistical weaknesses observed in most studies are discussed, and contributions are presented to improve the research in informatics in education.
\end{abstract}

Resumo. O Robomind é uma tecnologia educacional apoiada em recursos lúdicos e pedagógicos para dar suporte ao aprendizado de programação. Sumarizar as evidências acadêmicas já publicadas sobre o uso deste software é fundamental para avaliar sua eficácia. Uma revisão sistemática da literatura foi realizada e observou-se que os estudantes que utilizaram o Robomind apresentaram maior motivação com os estudos, interesse pela área de computação e menor evasão. Por outro lado, os ganhos de aprendizagem foram divergentes. Por fim, discutem-se as fragilidades científicas e estatísticas observadas na maioria dos estudos e contribuições são apresentadas para aperfeiçoar pesquisas na área de informática na educação.

\section{Introdução}

Muitos estudantes e educadores consideram o processo de ensino e aprendizagem de programação complexo [Qian and Lehman 2017]. O uso de tecnologias educacionais representa uma importante estratégia pedagógica para mitigar parte das dificuldades que ocorrem neste processo. Dentre as diversas opções disponíveis, o Robomind ${ }^{1}$ é um exemplo de ambiente lúdico e interativo para o estudante praticar os conceitos de programação.

Diversos estudos experimentais destinaram-se a avaliar os benefícios pedagógicos promovidos pelo Robomind. Sumarizar este corpo de conhecimento é fundamental para subsidiar a decisão dos professores em utilizar essa tecnologia educacional, bem como auxiliar pesquisadores sobre as lacunas existentes.

\footnotetext{
${ }^{1}$ https://www.robomind.net/pt/index.html
} 
Uma revisão sistemática da literatura foi realizada para alcançar o objetivo descrito acima. Sua realização centrou-se nas seguintes questões de pesquisa: Quais as contribuições pedagógicas que o Robomind proporcionou ao processo de ensino e aprendizagem? e b) Qual o conjunto de evidências sobre o uso do Robomind no processo de ensino e aprendizagem de programação?. Apresenta-se neste estudo a análise de 10 trabalhos que avaliaram a capacidade do Robomind em promover a motivação, aprendizado, interesse pela área de computação e na redução da evasão.

Este trabalho está organizado no seguinte formato: a Seção 2 apresenta a ferramenta Robomind e aborda-se as evidências científicas de outras tecnologias educacionais. Na Seção 3 detalha-se a metodologia utilizada nesta revisão sistemática, seguido dos resultados (Seção 4) e análise na Seção 5. Por fim, as limitações do estudo são apresentadas na Seção 6.

\section{Revisão da Literatura}

O Robomind tem por objetivo facilitar o aprendizado de programação por meio de uma narrativa lúdica que também contribui para a motivação dos estudantes. Algoritmos são utilizados para controlar um robô virtual em um ambiente interativo, dinâmico e intuitivo. O uso desse programa também contribui para a explanação de fundamentos da robótica, visto que contempla comandos básicos de um robô (e.g., visão/sensoriamento, movimento e interação com o ambiente). O desenvolvimento do programa foi guiado ao longo dos anos pelos pilares estabelecidos pelo Pensamento Computacional, contemplando desde a esfera de programação (e.g., variáveis, comandos, laços, condicionantes, funções e parâmetros) e matemática (álgebra e geometria) como também de resolução de problemas (dividir para conquistar) e aplicabilidade dos algoritmos em problemas reais (e.g., automação industrial, logística e transportes).

O uso de tecnologias educacionais, como o Robomind, pode promover diversos ganhos ao processo de ensino e aprendizagem, como o incentivo à motivação [Hamari et al. 2016], melhorias no aprendizado [Clark et al. 2016] e maior engajamento [Papastergiou 2009]. No entanto, são necessárias evidências científicas que comprovem os benefícios pedagógicos destas ferramentas [Bittencourt and Isotani 2018]. Este procedimento foi realizado com outras tecnologias educacionais, como o Scratch $^{2}$ e Alice ${ }^{3}$.

[Moreno-León and Robles 2016] apresentam uma revisão sistemática da literatura para avaliar a efetividade da ferramenta Scratch. Os autores identificaram que múltiplas habilidades são estimuladas com o seu uso e que podem contribuir em áreas como programação, matemática, física, ciências, entre outras. Falhas experimentais nos trabalhos citados foram identificadas e comprometeram a realização de uma meta-análise, como a ausência de grupos de controle e experimental e o uso de instrumentos não validação e/ou padronizados, dificuldades também encontrados por nós.

[Costa and Miranda 2017] também realizaram uma revisão sistemática, seguida de meta-análise para avaliar a eficácia da ferramenta Alice. Observou-se que o seu uso resultou em ganhos pedagógicos quando comparado ao ensino tradicional de programação. Os autores criticam o pequeno número de estudos experimentais disponíveis na literatura

\footnotetext{
${ }^{2}$ https://scratch.mit.edu/

${ }^{3}$ https://www.alice.org/
} 
IX Congresso Brasileiro de Informática na Educação (CBIE 2020)

Anais do XXXI Simpósio Brasileiro de Informática na Educação (SBIE 2020)

(seis estudos foram analisados), e destacaram falhas metodológicas que dificultaram o uso dos dados estatísticos para realização da meta-análise. Limitações que também estiveram presentes em nosso trabalho.

\section{Metodologia}

A condução da revisão sistemática da literatura segue a metodologia proposta em [Kitchenham 2004], em razão do seu alinhamento com a área de computação, e será detalhada nas seções subsequentes.

\subsection{Definição das Questões de Pesquisa}

Este estudo teve por objetivo sumarizar o corpo de evidências sobre os benefícios pedagógicos no uso do Robomind. Portanto, definiu-se as seguintes questões de pesquisa: Quais as contribuições pedagógicas que o Robomind proporcionou ao processo de ensino e aprendizagem? e b) Qual o conjunto de evidências sobre o uso do Robomind no processo de ensino e aprendizagem de programação?. Seguindo as diretrizes de [Jones et al. 1999], entende-se por contribuições pedagógicas: ganhos de aprendizagem (aquisição de conhecimento sobre um determinado tema), capacidade de motivar estudantes, como também o potencial em transformar (favoravelmente) opiniões sobre um determinado tema.

\subsection{Construção das Palavras-chave e Definição das Bases de Dados}

Optou-se pelo uso da palavra-chave robomind como termo de pesquisa nas bases de dados, pelo fato de representar o objeto investigado e por não ser uma palavra comum, reduzindo assim o número de ocorrências irrelevantes. Não foram definidas restrições de data de publicação nas buscas e foram analisados artigos publicados até o mês de junho de 2020.

Foram consideradas as bases de dados do IEEE, ACM, Eric, Science Direct, da Revista Brasileira de Informática na Educação (RBIE), Revista de Novas Tecnologias na Educação (RENOTE), Anais do Workshop de Informática na Escola (WIE), Workshop de Educação em Computação (WEI), Simpósio Brasileiro de Informática na Educação (SBIE) e o Catálogo de Teses e Dissertações da Capes. Essas bases são reconhecidas nacionalmente e internacionalmente na área de informática na educação. Em razão do pequeno número de resultados encontrados nessas bases, também foram realizadas pesquisas no Google Scholar, sendo que neste caso apenas trabalhos revisados por pares foram considerados.

\subsubsection{Critérios de Inclusão e Exclusão}

A inclusão dos trabalhos na revisão seguiu os seguintes critérios: a) o Robomind foi utilizado em um processo de intervenção educacional com estudantes; b) os estudantes foram avaliados sob um dos critérios definidos neste estudo como "contribuição pedagógica", relatados na seção 3.1; c) o conteúdo curricular da intervenção deveria abordar os conceitos sugeridos pelo currículo $\mathrm{ACM}^{4}$ na seção $\mathrm{SDF} / \mathrm{Conceitos}$ fundamentais de programação:

\footnotetext{
${ }^{4}$ https://www.acm.org/education/curricula-recommendations
} 
IX Congresso Brasileiro de Informática na Educação (CBIE 2020)

Anais do XXXI Simpósio Brasileiro de Informática na Educação (SBIE 2020)

Tabela 1. Relação dos trabalhos excluídos.

\begin{tabular}{|l|l|}
\hline Trabalho & Motivo \\
\hline [Major et al. 2014] & Não avaliava o Robomind \\
\hline [Ferreira et al. 2015] & $\begin{array}{l}\text { Não avaliou os ganhos pedagógicos do uso da ferramenta } \\
\text { com os estudantes. }\end{array}$ \\
\hline [Botelho et al. 2016] & Não envolvia o ensino de programação. \\
\hline [Santos et al. 2019] & $\begin{array}{l}\text { Não avaliou os ganhos pedagógicos do uso da ferramenta } \\
\text { com os estudantes; Utilizou múltiplas tecnologias educaci- } \\
\text { onais que impactam a avaliação individual dos ganhos do } \\
\text { Robomind. }\end{array}$ \\
\hline
\end{tabular}

variáveis, entrada/saída, condicionais, repetições e funções; e d) os estudos deveriam adotar algum instrumento para medir o efeito da intervenção. Os seguintes critérios de exclusão foram definidos: a) os artigos que não estivessem escritos em inglês, português ou espanhol; e b) que não estavam disponíveis para leitura completa.

\subsection{Critérios de Qualidade}

Para avaliar a qualidade dos estudos foram analisados critérios relevantes aos estudos experimentais na área de educação e descritos por [Fraenkel et al. 1993]: q1) questão de pesquisa estabelecida; q2) descrição de características da população sob intervenção; q3) descrição do contexto pedagógico; q4) especificação do desenho experimental; q5) uso de pré e pós-teste e q5) uso de grupo experimental e controle.

\section{Resultados}

Apenas as bases de dados do Google Scholar, ScienceDirect, SBIE, CBIE e WIE, retornaram resultados relevantes. 14 trabalhos foram selecionados na primeira etapa da análise. Na segunda etapa, em que houve a leitura completa dos estudos, dez se adequaram aos critérios de inclusão. Os quatro trabalhos excluídos e as respectivas razões estão descritas na Tabela 1.

Os trabalhos selecionados para leitura completa foram avaliados quanto aos critérios de qualidade definidos na Seção 3.3 e os resultados estão descritos na Tabela 2. Por fim, a avaliação completa dos trabalhos é detalhada na Tabela 3.

\section{1. a) Quais as contribuições pedagógicas que o Robomind proporciona ao processo de ensino e aprendizagem?}

Para responder essa questão de pesquisa, buscou-se compreender o foco de investigação dos estudos analisados. Observou-se que o Robomind foi avaliado sob quatro perspectivas: i) aprendizado de programação e resolução de problemas; ii) capacidade de motivar estudantes; iii) capacidade de proporcionar o interesse pela área de computação e iv) índices de evasão no componente curricular.

Os resultados de [Yuana and Maryono 2016], [Benitti et al. 2009] e [Conchinha et al. 2016] indicaram que os estudantes sentiram-se satisfeitos e motivados com as atividades desenvolvidas no Robomind. [Yuana and Maryono 2016], [Nofitasari et al. 2017], [Schoeffel et al. 2015] e [Maf'ulah et al. 2017] relataram ganhos 
IX Congresso Brasileiro de Informática na Educação (CBIE 2020)

Anais do XXXI Simpósio Brasileiro de Informática na Educação (SBIE 2020)

Tabela 2. Avaliação de qualidade.

\begin{tabular}{|l|l|l|l|l|l|l|}
\hline Trabalho & Q1 & Q2 & Q3 & Q4 & Q5 & Q6 \\
\hline [Yuana and Maryono 2016] & S & S & S & S & N & S \\
\hline [Nofitasari et al. 2017] & $\mathrm{N}$ & $\mathrm{N}$ & $\mathrm{S}$ & $\mathrm{N}$ & $\mathrm{N}$ & $\mathrm{N}$ \\
\hline [Schoeffel et al. 2015] & $\mathrm{N}$ & $\mathrm{S}$ & $\mathrm{S}$ & $\mathrm{N}$ & $\mathrm{N}$ & $\mathrm{N}$ \\
\hline [Maf'ulah et al. 2017] & $\mathrm{N}$ & $\mathrm{N}$ & $\mathrm{N}$ & $\mathrm{S}$ & $\mathrm{S}$ & $\mathrm{S}$ \\
\hline [Durães 2015] & $\mathrm{N}$ & $\mathrm{S}$ & $\mathrm{S}$ & $\mathrm{N}$ & $\mathrm{S}$ & $\mathrm{S}$ \\
\hline [Benitti et al. 2009] & $\mathrm{N}$ & $\mathrm{S}$ & $\mathrm{S}$ & $\mathrm{N}$ & $\mathrm{S}$ & $\mathrm{N}$ \\
\hline [Koorsse et al. 2015] & $\mathrm{S}$ & $\mathrm{N}$ & $\mathrm{S}$ & $\mathrm{S}$ & $\mathrm{S}$ & $\mathrm{S}$ \\
\hline [Conchinha et al. 2016] & $\mathrm{N}$ & $\mathrm{S}$ & $\mathrm{S}$ & $\mathrm{N}$ & $\mathrm{N}$ & $\mathrm{N}$ \\
\hline [Faisal et al. 2017] & $\mathrm{S}$ & $\mathrm{N}$ & $\mathrm{S}$ & $\mathrm{S}$ & $\mathrm{S}$ & $\mathrm{S}$ \\
\hline [Soares et al. 2018] & $\mathrm{S}$ & $\mathrm{S}$ & $\mathrm{S}$ & $\mathrm{S}$ & $\mathrm{N}$ & $\mathrm{S}$ \\
\hline
\end{tabular}

no aprendizado de programação com o uso do Robomind em múltiplos níveis educacionais. [Durães 2015] destaca os resultados positivos no ensino de STEM, contemplando física e matemática, para além da programação. [Koorsse et al. 2015], comparou o uso do Robomind, Scratch e B\# no aprendizado de programação e os resultados de aprendizagem foram similares, com exceção dos conceitos de estrutura de repetição em que o Robomind se favoreceu. No estudo [Faisal et al. 2017] o Robomind também foi comparado ao Scratch, e os autores identificaram que o uso do Scratch promoveu maiores ganhos para o aprendizado. [Soares et al. 2018] compararam os desempenhos acadêmicos de estudantes que utilizaram o Robomind com outros estudantes em que o professor utilizou uma metodologia tradicional de ensino (sem uso de tecnologias educacionais e com uma metodologia majoritariamente expositiva). Não foram identificadas diferenças estatisticamente significantes no número de aprovados no componente curricular, mas o grupo que utilizou o Robomind apresentou menor evasão.

\section{2. b) Qual o conjunto de evidências sobre o uso do Robomind no processo de ensino e aprendizagem de programação?}

Para sumarizar o corpo de evidências apontado na questão de pesquisa ii), realizou-se um procedimento estatístico de meta-análise que consiste na sumarização dos resultados de múltiplos estudos [Cohen et al. 2013]. Apenas um estudo apresentou o tamanho de efeito (effect-size) [Soares et al. 2018], informação necessária ao cálculo da meta-análise. Optou-se então por realizar o cálculo para os demais estudos que deveriam seguir os critérios: i) presença de um grupo de controle; ii) apresentar a média e desvio padrão dos grupos e iii) a variável dependente dos experimentos mensurem informações que façam sentido de serem comparadas.

Os estudos [Yuana and Maryono 2016], [Nofitasari et al. 2017], [Schoeffel et al. 2015], [Conchinha et al. 2016] e [Benitti et al. 2009] não utilizaram grupo controle e foram imediatamente descartados. Os trabalhos [Durães 2015] e [Maf'ulah et al. 2017] não apresentaram os dados estatísticos necessários para cálculo do tamanho de efeito. Apenas o estudo [Koorsse et al. 2015] contemplou os dois primeiros requisitos, no entanto, observou-se uma diferença significativa nas variáveis dependentes quando comparado ao estudo [Soares et al. 2018], o que não possibilitou a realização do procedimento de meta-análise. 


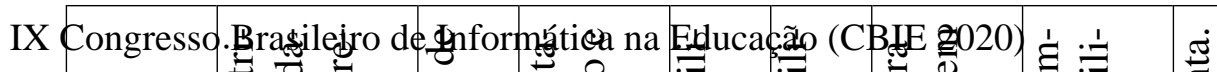

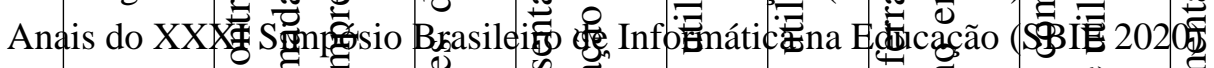

\begin{tabular}{|c|c|c|c|c|c|c|c|c|c|c|}
\hline 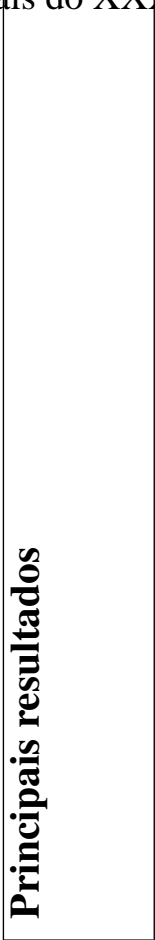 & 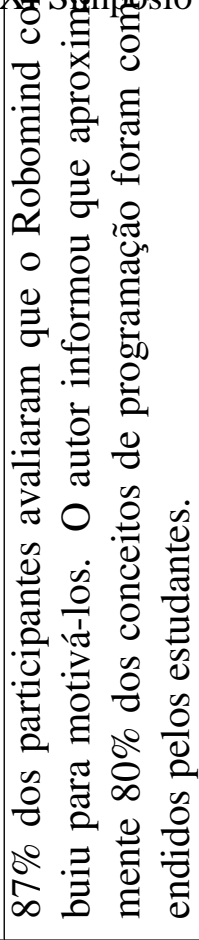 & 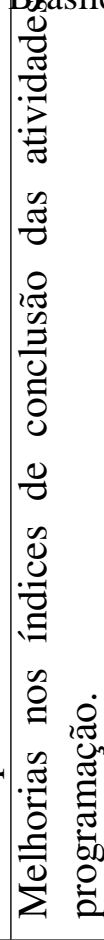 & 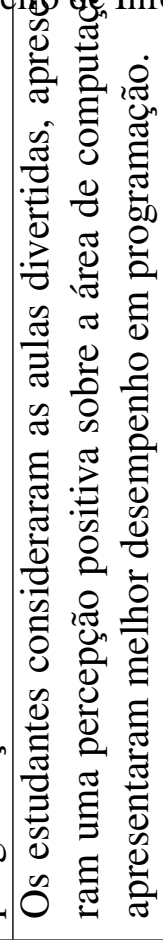 & 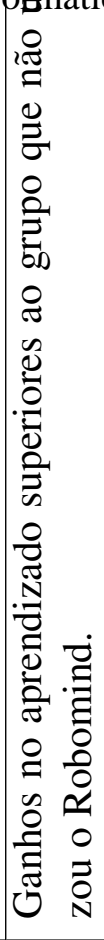 & 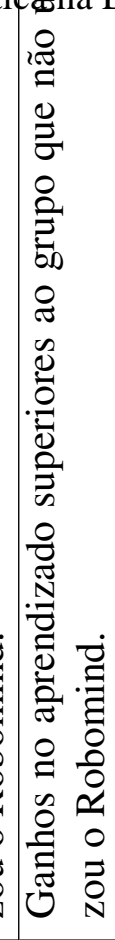 & 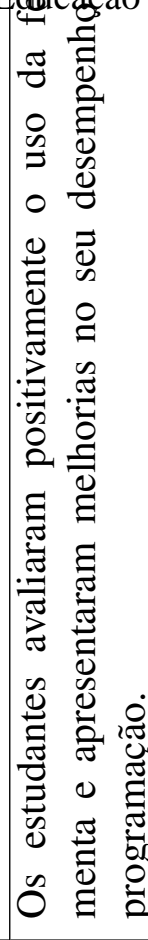 & 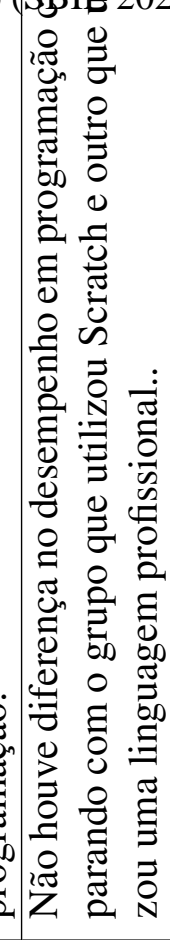 & 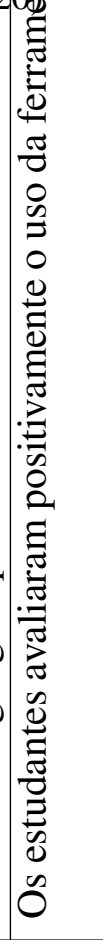 & 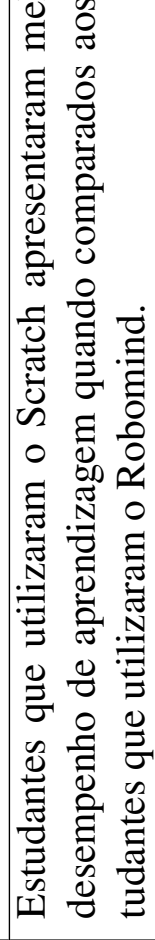 & 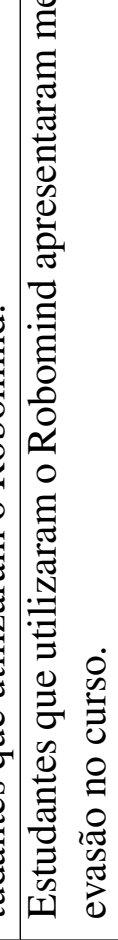 \\
\hline 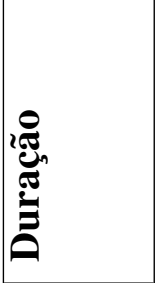 & 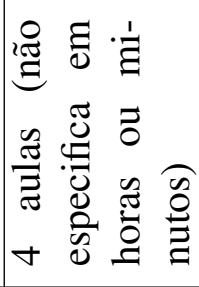 & 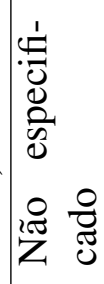 & 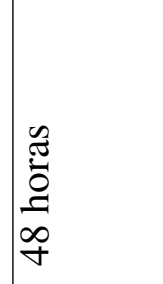 & $\begin{array}{l}0 \\
0 \\
0 \\
\Xi \\
0 \\
0\end{array}$ & 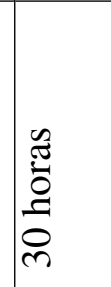 & 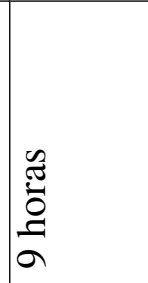 & $\begin{array}{l}\tilde{d} \\
\mathscr{d} \\
\Xi \\
\Xi \\
=\end{array}$ & 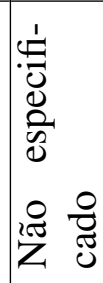 & 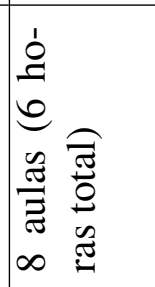 & 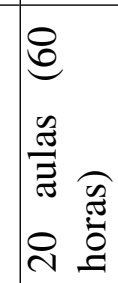 \\
\hline 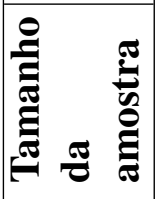 & $\varepsilon$ & $m$ & mे & $\tilde{6}$ & શे & a & $\frac{0}{2}$ & 2 & $g$ & 6 \\
\hline 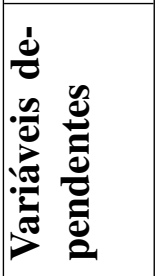 & 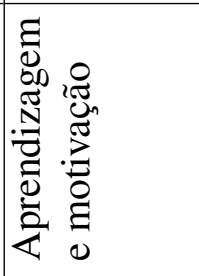 & 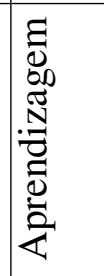 & 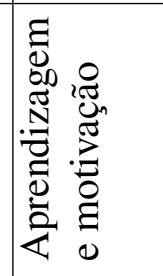 & 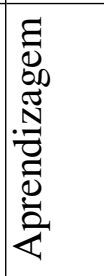 & 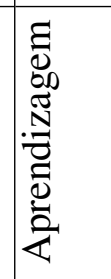 & 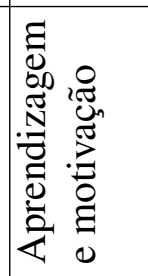 & 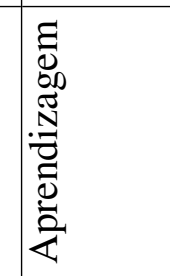 & 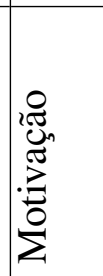 & 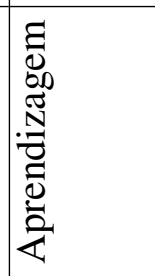 & 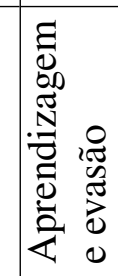 \\
\hline 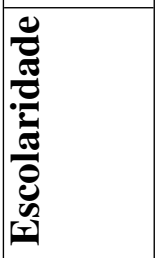 & 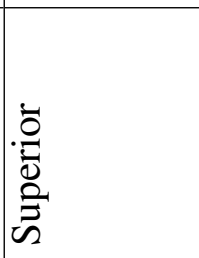 & 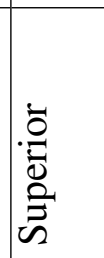 & 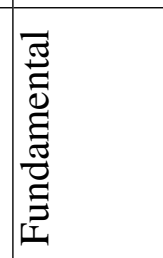 & 㝘 & 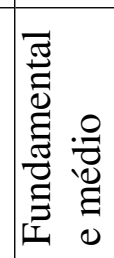 & $\begin{array}{l}: 0 \\
\sum_{0}^{0} \\
\sum\end{array}$ & 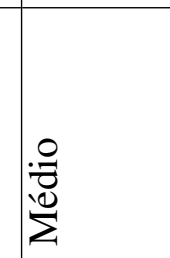 & 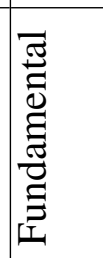 & $\begin{array}{l}\stackrel{0}{\Xi} \\
\sum_{\Sigma}^{0}\end{array}$ & 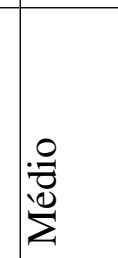 \\
\hline 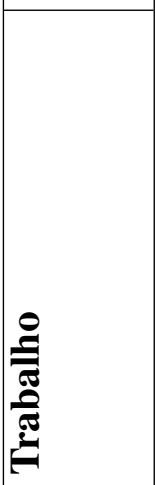 & 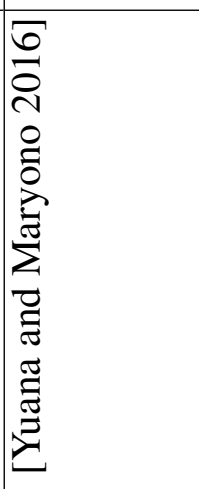 & 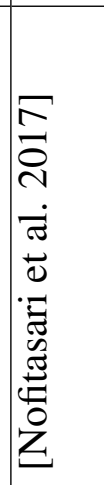 & 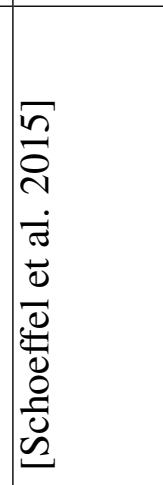 & 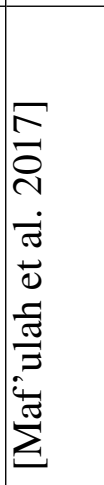 & 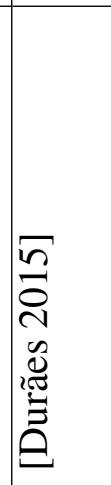 & 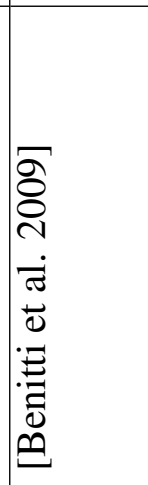 & 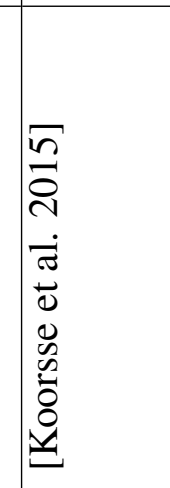 & 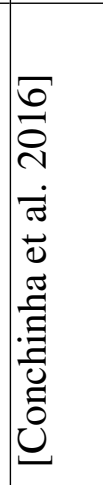 & 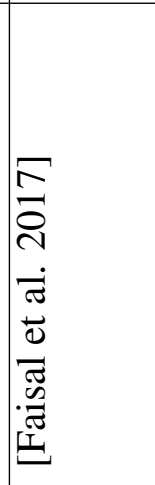 & 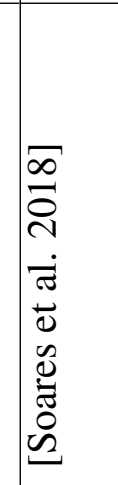 \\
\hline
\end{tabular}


IX Congresso Brasileiro de Informática na Educação (CBIE 2020)

Anais do XXXI Simpósio Brasileiro de Informática na Educação (SBIE 2020)

\section{Análise dos Resultados}

As evidências sugerem que o Robomind ofereceu suporte motivacional aos estudantes de programação que se engajaram nas atividades e sentiram-se satisfeitos com sua realização. Isto é relevante, pois a motivação é um aspecto fundamental ao aprendizado, em especial de programação [Gomes et al. 2018]. No entanto, os trabalhos que analisaram o construto motivacional não utilizaram instrumentos padronizados e confiáveis. Isso representa uma grande ameaça à validade dos resultados, uma vez que não é garantida a confiabilidade dos instrumentos adotados. Para evitar este tipo de ameaça, sugere-se que trabalhos futuros utilizem instrumentos já consolidados e validados cientificamente.

Os resultados sobre os ganhos acadêmicos oferecidos pelo uso do Robomind precisam ser ponderados, pois em alguns casos são conflitantes. Além disso, há falhas experimentais que comprometem sua interpretação. Por exemplo, [Yuana and Maryono 2016], [Nofitasari et al. 2017], [Schoeffel et al. 2015], [Benitti et al. 2009] e [Conchinha et al. 2016] não utilizaram grupo controle para comparar os resultados, o que reduz a capacidade de avaliação se os resultados encontrados podem ser atribuídos à ferramenta ou a outro fator. Outra falha refere-se à ausência de testes estatísticos. Por exemplo, [Durães 2015] e [Nofitasari et al. 2017] não realizaram esse cálculo para avaliar se as diferenças encontradas nos resultados da aplicação do pré e pós-teste são estatisticamente significantes. Em [Schoeffel et al. 2015], os estudantes que obtiveram melhores resultados também utilizaram outras ferramentas de programação durante a intervenção, como o Scratch, tornando difícil mensurar se as contribuições ao aprendizado foram em razão do uso do Robomind, Scratch ou da combinação de ambos. Também houve resultados que não foram favoráveis ao Robomind, ao compará-lo com metodologias tradicionais de ensino ou outros ambientes educativos, como o Scratch. Portanto, sugere-se que novos estudos experimentais sejam realizados para construir um corpo de evidência sobre este tema, em especial com o uso de grupos controle para oferecer assim maior validade científica.

Apesar das evidências sobre os ganhos de aprendizagem com o uso do Robomind serem inconclusivas, ainda sim não se observou perdas quando comparado ao ensino tradicional. Ou seja, utilizar ou não essa ferramenta teria o mesmo efeito. No entanto, há indicativos da sua efetividade em motivar estudantes e na redução de evasão.

\subsection{Críticas aos Procedimentos Metodológicos dos Estudos}

Esta seção é dedicada à críticas acerca dos procedimentos científicos, experimentais e estatísticos, dos estudos avaliados. Como observamos que estas críticas também se fazem presentes em outros estudos da área de aprendizagem de programação, uma reflexão é proposta como forma de melhorar a qualidade de estudos futuros.

Há na literatura um quantitativo significativo de ferramentas que se propõem a apoiar o processo de ensino e aprendizagem de programação. Aparentemente a facilidade em desenvolver essas ferramentas não é a mesma com a qual elas são avaliadas empiricamente. Isto se reflete nas dificuldades reportadas por revisões que avaliaram tecnologias educacionais conhecidas, como o Scratch e Alice [Costa and Miranda 2017]; [Moreno-León and Robles 2016], e também em nosso estudo. Isto é prejudicial, pois pode-se adotar estratégias pedagógicas ineficazes para a educação, ou até mesmo em casos mais graves, elas podem prejudicar o processo de ensino e aprendizagem. 
Também foram encontrados graves problemas no delineamento de experimentos e cálculos estatísticos da maioria dos estudos identificados. Por exemplo, falta de grupo controle, ausência de aleatorização das amostras, não realização de pré e pós-testes, e uso de instrumentos não padronizados e validados. Melhorias são necessárias para reduzir as ameaças à validade interna e externa dos experimentos. Em razão destes problemas, a generalização dos resultados e verificação de causalidade, que são princípios básicos de um experimento, são fortemente ameaçados. Entende-se que há a necessidade de promover formação aos pesquisadores acerca dos procedimentos científicos e estatísticos para aperfeiçoar a qualidade dos estudos e reduzir as ameaças à validade de seus experimentos.

\section{Limitações do estudo}

Para identificar as ameaças à validade deste estudo, utilizou-se a relação de ameaças para revisões na área de computação proposta por [Zhou et al. 2016]. Duas ameaças foram observadas: i) o pequeno quantitativo de resultados identificados e ii) o viés na seleção de estudos. Este último, em razão de uma das etapas do processo de seleção dos trabalhos ter sido conduzida por apenas um pesquisador, haja visto que alguns trabalhos podem ter sido excluídos de forma inadequada.

\section{Conclusão}

Uma revisão sistemática da literatura foi realizada com o objetivo de identificar evidências pedagógicas sobre o uso da ferramenta Robomind no contexto do ensino de programação. Os estudantes que utilizaram essa ferramenta apresentaram maior motivação e menor evasão, mas os relatos sobre ganhos de aprendizagem são conflitantes. Apesar das frágeis evidências, o Robomind apresenta potencial para ser utilizado no ensino de programação. No entanto, novos estudos são necessários para construir um corpo de evidências robusto sobre essa temática.

Falhas nos procedimentos científicos e experimentais dos estudos analisados limitaram a interpretação dos seus resultados. Sugestões de aperfeiçoamento metodológico foram apresentadas visando melhorias científicas nos estudos sobre informática na educação.

\section{Referências}

Benitti, F. B. V., Vahldick, A., Urban, D. L., Krueger, M. L., and Halma, A. (2009). Experimentação com robótica educativa no ensino médio: ambiente, atividades e resultados. In Anais do Workshop de Informática na Escola, volume 1, pages 1811-1820.

Bittencourt, I. I. and Isotani, S. (2018). Informática na educação baseada em evidências: Um manifesto. Revista Brasileira de Informática na Educação, 26(03):108.

Botelho, W. T., Marietto, M. d. G. B., Ferreira, J. C. d. M., and Pimentel, E. P. (2016). Kolb's experiential learning theory and belhot's learning cycle guiding the use of computer simulation in engineering education: A pedagogical proposal to shift toward an experiential pedagogy. Computer Applications in Engineering Education, 24(1):7988.

Clark, D. B., Tanner-Smith, E. E., and Killingsworth, S. S. (2016). Digital games, design, and learning: A systematic review and meta-analysis. Review of educational research, 86(1):79-122. 
IX Congresso Brasileiro de Informática na Educação (CBIE 2020)

Anais do XXXI Simpósio Brasileiro de Informática na Educação (SBIE 2020)

Cohen, L., Manion, L., and Morrison, K. (2013). Research methods in education. routledge.

Conchinha, C., Leal, M., and de Freitas, J. C. (2016). Robots \& nee: A robótica virtual como promotora de inclusão e da aprendizagem por projetos lúdicos robots \& nee: Virtual robotics as a promoter for inclusion and learning by playful projects. In $I V$ Conferência Ibérica de Inovação na Educação com TIC.

Costa, J. M. and Miranda, G. L. (2017). Relation between alice software and programming learning: A systematic review of the literature and meta-analysis. British Journal of Educational Technology, 48(6):1464-1474.

Durães, D. A. (2015). Gaming and robotics to transforming learning. In Methodologies and Intelligent Systems for Technology Enhanced Learning, pages 51-56. Springer.

Faisal, M., Yuana, R., and Basori, M. (2017). Comparative study between robomind and scratch as programming assistance tool in improving understanding of the basic programming concepts. In International Conference on Teacher Training and Education 2017 (ICTTE 2017). Atlantis Press.

Ferreira, M., de Lima, J., Santos, H., Oliveira, E., and do Nascimento, J. (2015). Computação para ensino médio na modalidade semipresencial: Uma experiência da disciplina de estágio supervisionado. In Anais do XXIII Workshop sobre Educação em Computação, pages 406-415. SBC.

Fraenkel, J. R., Wallen, N. E., and Hyun, H. H. (1993). How to design and evaluate research in education, volume 7. McGraw-Hill New York.

Gomes, A., Ke, W., Lam, C.-T., Marcelino, M. J., and Mendes, A. (2018). Student motivation towards learning to program. In 2018 IEEE Frontiers in Education Conference (FIE). IEEE.

Hamari, J., Shernoff, D. J., Rowe, E., Coller, B., Asbell-Clarke, J., and Edwards, T. (2016). Challenging games help students learn: An empirical study on engagement, flow and immersion in game-based learning. Computers in human behavior, 54:170179.

Jones, A., Scanlon, E., Tosunoglu, C., Morris, E., Ross, S., Butcher, P., and Greenberg, J. (1999). Contexts for evaluating educational software. Interacting with computers, 11(5):499-516.

Kitchenham, B. (2004). Procedures for performing systematic reviews. Keele, UK, Keele University, 33(2004):1-26.

Koorsse, M., Cilliers, C., and Calitz, A. (2015). Programming assistance tools to support the learning of it programming in south african secondary schools. Computers \& Education, 82:162-178.

Maf'ulah, I. A., Efendi, A., and Basori, B. (2017). The effectiveness of robomind software in basic programming learning. IJIE (Indonesian Journal of Informatics Education), $1(1): 30-37$.

Major, L. et al. (2014). An empirical investigation into the effectiveness of a robot simulator as a tool to support the learning of introductory programming. $\mathrm{PhD}$ thesis, Keele University. 
Moreno-León, J. and Robles, G. (2016). Code to learn with scratch? a systematic literature review. In 2016 IEEE Global Engineering Education Conference (EDUCON), pages 150-156. IEEE.

Nofitasari, A., Yuana, R. A., and Maryono, D. (2017). The use of robomind application in problem based learning model to enhance the student's understanding on the conceptual programming algorithm. IJIE (Indonesian Journal of Informatics Education), 1(1):9-18.

Papastergiou, M. (2009). Digital game-based learning in high school computer science education: Impact on educational effectiveness and student motivation. Computers \& education, 52(1):1-12.

Qian, Y. and Lehman, J. (2017). Students' misconceptions and other difficulties in introductory programming: A literature review. ACM Transactions on Computing Education (TOCE), 18(1):1-24.

Santos, H., Souza, F., Santos, B., Amorim, I., Silva, M., et al. (2019). Ensino de introdução à programação na modalidade semipresencial com o apoio do google classroom. In Anais do XXVII Workshop sobre Educação em Computação, pages 131-140. SBC.

Schoeffel, P., Moser, P., Varela, G., Durigon, L., de Albuquerque, G. C., and Niquelatti, M. (2015). Uma experiência no ensino de pensamento computacional para alunos do ensino fundamental. In Anais dos Workshops do Congresso Brasileiro de Informática na Educação, volume 4, page 1474.

Soares, L., Cavalcanti, E. R., et al. (2018). Avaliaçao experimental do robomind no ensino de programaçao com estudantes do curso técnico em informática integrado ao ensino médio. In Brazilian Symposium on Computers in Education (Simpósio Brasileiro de Informática na Educação-SBIE), volume 29, page 288.

Yuana, R. A. and Maryono, D. (2016). Robomind utilization to improve student motivation and concept in learning programming. In Proceeding of International Conference on Teacher Training and Education, volume 1, pages 962-966.

Zhou, X., Jin, Y., Zhang, H., Li, S., and Huang, X. (2016). A map of threats to validity of systematic literature reviews in software engineering. In 2016 23rd Asia-Pacific Software Engineering Conference (APSEC), pages 153-160. IEEE. 